\title{
Short-distance wavefunction statistics in one-dimensional Anderson localization
}

\author{
H. Schomerus ${ }^{\mathrm{a}}$ and M. Titov \\ Max-Planck-Institut für Physik komplexer Systeme, Nöthnitzer Str. 38, 01187 Dresden, Germany
}

Received 10 July 2003

Published online 15 October 2003 - (c) EDP Sciences, Società Italiana di Fisica, Springer-Verlag 2003

\begin{abstract}
We investigate the short-distance statistics of the local density of states $\nu$ in long one-dimensional disordered systems, which display Anderson localization. It is shown that the probability distribution function $P(\nu)$ can be recovered from the long-distance wavefunction statistics, if one also uses parameters that are irrelevant from the perspective of two-parameter scaling theory.
\end{abstract}

PACS. 72.15.Rn Localization effects (Anderson or weak localization) - 05.40.-a Fluctuation phenomena, random processes, noise, and Brownian motion - 42.25.Dd Wave propagation in random media -

73.20.Fz Weak or Anderson localization

\section{Introduction}

Wave localization in a disordered potential is the most striking hallmark of systematic interference by multiple coherent scattering [1-8]. Systematic constructive interference in a spatially localized region results in a confinement of the wavefunction, which decays exponentially away from the localization center (with a decay length $l_{\text {loc }}$, the localization length), in contrast to the extended waves in constant or spatially periodic potentials. Localization comes along with large fluctuations of the wavefunction, which can be induced by changing the disorder configuration. The wavefunction statistics can be probed, e.g., globally across a system of finite length $L_{\text {sys }}$ by the dimensionless conductance (transmission probability) $g$, and inside a semi-infinite system $\left(L_{\mathrm{sys}}=\infty\right)$ by the local density of states $\nu$ at a distance $L_{\text {open }}$ to the opening.

Theories of localization often focus on the longdistance wavefunction statistics, where a high degree of universality prevails. For instance, distribution functions are restricted to log-normal forms as a consequence of the central-limit theorem, which leads to two-parameter scaling (TPS) [9]. Consequentially, for the local density of states, the probability distribution function $P(\nu)$ is characterized by the mean $\operatorname{logarithm} C_{1}^{(\nu)} \equiv-\langle\ln \nu\rangle$ and its variance $C_{2}^{(\nu)} \equiv \operatorname{var} \ln \nu$. The TPS observation has found many applications [10-16]. An even enhanced degree of universality arises in the random-phase approximation (RPA), where single-parameter scaling (SPS) applies [17-20], and both parameters further are connected,

\footnotetext{
a e-mail: henning@mpipks-dresden.mpg.de
}

e.g. by $C_{2}^{(\nu)} \sim 2 C_{1}^{(\nu)}$ for one-dimensional systems [21-23]. (It was recognized very early that SPS breaks down for strong disorder, see, e.g., Ref. [24].)

In this paper we point out a connection of the longdistance statistics to the short-distance statistics in the one-dimensional Anderson model of localization, probed by the local density of states $\nu$. Namely, we find that the distribution function $P(\nu)$ for short distances reliably can be approximated with the help of parameters that are extracted from the long-distance limit, including parameters (besides $C_{1}^{(\nu)}$ and $C_{2}^{(\nu)}$ ) that are irrelevant, from the perspective TPS, for the long-distance wavefunction statistics themselves.

We start this paper by an analysis of $P(\nu)$ and $P(g)$ within the concepts of large-deviation statistics [25], which goes beyond the central-limit theorem, and identify quantities $C_{n}, n \geq 3$, which are irrelevant for the longdistance wavefunction statistics, but will turn out to be useful for the short-distance wavefunction statistics. Each quantity defines its own length scale by its asymptotic slope $c_{n}=\lim _{L \rightarrow \infty} d C_{n} / d L$ (where $L \equiv L_{\text {sys }}$ for $g$ and $L \equiv L_{\text {open }}$ for $\nu$ ), in analogy to the relation between $C_{1} \sim 2 L / l_{\text {loc }}$ and the localization length $l_{\text {loc }}$. The length scales obtained from $\nu$ and $g$ coincide. The constant offsets $d_{n}=\lim _{L \rightarrow \infty} C_{n}-L c_{n}$ are shown to contain information on the reflection phase, which allows to test the RPA.

Then we discuss that $P(\nu)$ for short distances $L_{\text {open }} \lesssim$ $l_{\text {loc }}$ can be reconstructed from the parameters $c_{n}$ and $d_{n}$. This is in striking contrast to $P(g)$, for which the parameters show a transient behavior for small $L_{\text {sys }}$ (where wavefunctions are not yet localized), as was pointed out very recently in reference [13] (see also Ref. [26]). Our 
observations for the short-distance statistics lead us to conclude that the cumulants $C_{n}$ are useful characteristics of localized wavefunctions, even though they are not relevant in the long-distance limit because of TPS.

Finally, we analytically and numerically investigate the parameters $c_{n}$ and $d_{n}$ in various regimes of the onedimensional Anderson model.

The paper is organized as follows: In Section 2 we describe the general implications of large-deviation statistics for the scaling of the distribution functions $P(g)$ and $P(\nu)$, and identify the parameters $c_{n}$ and $d_{n}$ in the cumulants $C_{n}, n \geq 3$. In Section 3 we specialize to the onedimensional Anderson model. In order to motivate subsequent considerations, we first illustrate in Section 3.1 the length dependence of the cumulants $C_{n}$ by numerical simulations. Then (Sect. 3.2) we briefly review the analytical theory for the asymptotic slopes $c_{n}[26,27]$ and extent it to the case of competition between onsite disorder and offsite disorder close to the band center. We also present the theory for the asymptotic offsets $d_{n}$. In Section 3.3 we investigate the dependence of the parameters in various regimes of the Anderson model. Our conclusions are given in Section 4.

In order to facilitate a parallel discussion of the statistics of $g$ and $\nu$, we use the common notation $L \equiv$ $L_{\text {sys }}$ when considering $g$ and $L \equiv L_{\text {open }}$ when considering $\nu$. One has to bear in mind that in the latter case, $L_{\text {sys }}=\infty$ and hence one always discusses localized wavefunctions, while in the former case this is true only for $L \equiv L_{\mathrm{sys}} \gg l_{\mathrm{loc}}$.

\section{Large-deviation statistics}

Large-deviation statistics often is introduced as the third and final step in a progressively refined analysis of the asymptotic behavior of probability distribution functions, where the first step is the law of large numbers and the second is the central-limit theorem. In localization, the law of large numbers certifies that the Lyapunov exponent $\gamma=C_{1}^{(g)} / 2 L$ is self-averaging in the limit $L \rightarrow \infty[28]$, with asymptotic value $\lim _{L \rightarrow \infty} \gamma=l_{\text {loc }}^{-1}$. The central-limit theorem delivers a statement about the finite-length corrections to this asymptotic value, which are characterized by $C_{2}^{(g)}$ : The variance var $\gamma=C_{2}^{(g)} / L^{2}$ decreases asymptotically as $L^{-1}$. Presently, we find it useful not address the Lyapunov exponents, since these are defined with help of the system length $L$, but to rely on quantities that only involve $g$ or $\nu$, like $C_{1}^{(g)}, C_{2}^{(g)}, C_{1}^{(\nu)}$, and $C_{2}^{(\nu)}$. The law of large numbers and the central-limit theorem predict a linear growth of these quantities with $L$. The full picture is unfolded in the framework of large-deviation statistics [25]: All cumulants can increase linearly with length or distance,

$$
\begin{aligned}
& C_{n}^{(g)}=\left\langle\left\langle(-\ln g)^{n}\right\rangle\right\rangle \sim c_{n}^{(g)} L+d_{n}^{(g)} \quad\left(L \gg l_{\mathrm{loc}}\right), \\
& C_{n}^{(\nu)}=\left\langle\left\langle(-\ln \nu)^{n}\right\rangle\right\rangle \sim c_{n}^{(\nu)} L+d_{n}^{(\nu)} \quad\left(L \gg l_{\mathrm{loc}}\right),
\end{aligned}
$$

where the coefficients $d_{n}$ are the subleading corrections that can be neglected in the asymptotic limit, but will be seen to encode information on the reflection phase that allows to test the validity of the RPA. For the conductance $g$, the linear scaling of the cumulants $C_{n}^{(g)}$ with $L$ and the connection of the $d_{n}^{(g)}$ to reflection phases also has been found in a constructive theory by Roberts [29]. The parameters $c_{n}$ can be extracted from the averages

$$
c^{(g)}(\xi)=-\lim _{L \rightarrow \infty} \frac{1}{L} \ln \left\langle g^{-\xi}\right\rangle=\sum_{n} \frac{\xi^{n}}{n !} c_{n}^{(g)}
$$

(or equivalently for $\nu$ ) as function of the continuous parameter $\xi$. Note the exponential dependence of the moments on $L$ due to localization, in contrast to the power-law dependence in the critical regime around a metal-insulator transition [7].

This paper is centered around our numerical observation in Section 3 that equation (1b) holds even for short distances to the opening $L_{\text {open }} \lesssim l_{\text {loc }}$, and hence can be used in regions where the central-limit theorem does not apply. This makes the parameters $c_{n}$ and $d_{n}$ with $n \geq 3$ observable in the distribution function $P(\nu)$, while in the long-distance behavior only $c_{1}$ and $c_{2}$ are relevant parameters [9].

Presently, analytical results for the distribution function $P(g)$ and $P(\nu)$ for short distances are only available in the regime of single-parameter scaling. The local density of states obeys a strict log-normal distribution for all distances $[23,30]$, and hence complies with our central observation. Equation (1a) cannot be extended to short distances, even in the regime of single-parameter scaling [21]; for studies outside this regime see, e.g., references $[13,26]$.

\section{One-dimensional Anderson model}

The previous Section 2 put forward some very general arguments from large-deviation statistics. The relevance of the asymptotically defined parameters $c_{n}$ (and $\left.d_{n}\right), n \geq 3$ for finite-distance wavefunction statistics, and the question whether these parameters indeed contain information that is independent from what is encoded in the parameters $c_{1}$ and $c_{2}$, only can be answered by a direct investigation. In the following we analyze the wavefunction statistics in the one-dimensional Anderson model [3], given by the Schrödinger equation discretized on a chain (lattice constant $a \equiv 1$ )

$$
t_{l-1} \psi_{l-1}+t_{l} \psi_{l+1}=\left(V_{l}-E\right) \psi_{l}
$$

where the hopping matrix elements $t_{l}$ and the disorder potential $V_{l}$ are random. We assume box distributions with $\left\langle t_{l}\right\rangle=t,\left\langle V_{l}\right\rangle=0,\left\langle t_{l} t_{m}\right\rangle=t^{2}+\frac{1}{2} D_{t} \delta_{l m}$, and $\left\langle V_{l} V_{m}\right\rangle=2 D_{V} \delta_{l m}$. Without any restriction we can set $t=1$, which fixes the energy scale in the dispersion relation $E(k)=-2 \cos k$ of the clean system $\left(D_{t}=D_{V}=0\right)$. The disorder strength will be characterized by the perturbative mean-free path [31]

$$
l_{\text {pert }}=\left(4-E^{2}\right) /\left(D_{V}+D_{t}\right),
$$


and the balance between onsite and offsite disorder will be characterized by the parameter

$$
\delta=\left(D_{V}-D_{t}\right) /\left(D_{V}+D_{t}\right)
$$

First we present the results of numerical simulations to illustrate the usefulness of the cumulants $C_{n}$. Next, in order to give a flavor for the mechanism behind the asymptotic linear growth (1) of the cumulants for the specific case of wavefunction localization, we extent the analytical theory of references $[26,27]$ for the asymptotic slopes $c_{n}$ to the case of competition of onsite- and offsite disorder close to the band center $E=0$, and also present the theory for the offsets $d_{n}$. The extension equips us with a means to violate SPS, which is finally compared to other means in order to determine the mutual (in)dependence of the parameters $c_{n}$ and $d_{n}$.

\subsection{Numerical illustration of the cumulants $C_{n}$}

Here we illustrate the length dependence of the cumulants $C_{n}$ by the results of numerical computations in ensembles of $10^{6}-10^{8}$ disorder realizations. The results for different strengths of onsite disorder $(\delta=1)$ are presented in Figure $1\left(l_{\text {pert }}=300\right)$, Figure $2\left(l_{\text {pert }}=24\right)$, and Figure $3\left(l_{\text {pert }}=1.5\right)$. Three representative values of energy are chosen: (a) $E=0$ at the band center, (b) $E=-1$ in the SPS region, (c) $E=-1.95$ close to the band edge. [The constant perturbative mean free path $l_{\text {pert }}$ in any figure has been obtained by adjusting the disorder strength according to equation (4); for values see the figure captions.] The significance of these three regions of energy will be discussed in the following Section 3.2. Plotted as a function of length are the cumulants $C_{n}$ calculated from $g$ and $\nu$, as well as from the 'mesoscopic' local density of states $\tilde{\nu}$, which is obtained from $\nu$ by averaging over a Fermi wavelength $\lambda_{\mathrm{F}}=2 \pi / \arccos (-E / 2)$ (with $\lambda_{\mathrm{F}}=4$ for $E=0, \lambda_{\mathrm{F}}=6$ for $E=-1$, and $\lambda_{F} \approx 28$ for $E=-1.95)$. The mesoscopic density of states accounts for a limited resolution that may be encountered in an experiment. It discards the nodes of the wavefunction (whose impact strongly depends on the dimensionality of the system) and only captures the smoothly varying envelope (which is more robust).

The cumulants all increase linearly for $L \gg l_{\text {loc }}$, and one may associate a length scale $\lim _{L \rightarrow \infty} 2 L / C_{n}=2 / c_{n}$ to each of them. The slopes $c_{n}$ are identical for all three underlying objects, and hence the sets of parameters $\left\{c_{n}^{(g)}\right\}=\left\{c_{n}^{(\nu)}\right\}=\left\{c_{n}^{(\tilde{\nu})}\right\} \equiv\left\{c_{n}\right\}$ coincide.

As advertised above, the cumulants $C_{n}^{(\nu)}$ and $C_{n}^{(\tilde{\nu})}$ increase linearly already for small $L \lesssim l_{\text {loc }}$ (moreover, the offsets for $\tilde{\nu}$ are vanishingly small), while the cumulants $C_{n}^{(g)}$ become linear only after some transient length, see reference [13] (these cumulants also have a finite offset $\left.d_{n}^{(g)}\right)$. This means that the asymptotically defined parameters $c_{n}^{(\nu)}$ and $d_{n}^{(\nu)}$ can be used to estimate the shortdistance behavior of $C_{n}^{(\nu)}$ and $C_{n}^{(\tilde{\nu})}$. In order to estimate the distribution functions, parameters with $n \geq 3$ have to

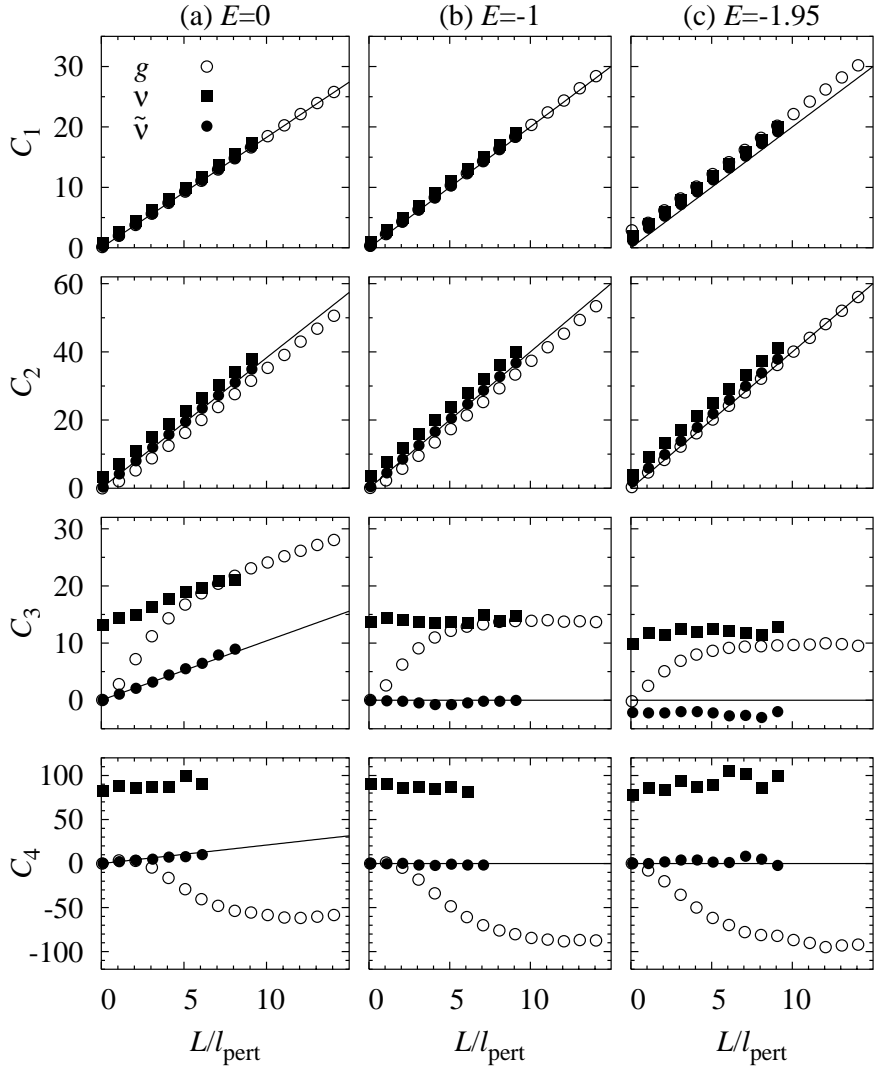

Fig. 1. Cumulants $C_{n}$ calculated from $g$ as a function of $L \equiv$ $L_{\text {sys }}$, and from $\nu, \tilde{\nu}$ as a function of $L \equiv L_{\text {open }}$. The data points are obtained by a numerical simulation of the one-dimensional Anderson model with onsite disorder, for $E=0, D_{V}=1 / 75$, (left panels), $E=-1, D_{V}=1 / 100$ (middle panels), and $E=-1.95, D_{V}=0.0006583$ (right panels). This corresponds to weak disorder, with a perturbative mean-free path of $l_{\text {pert }}=$ 300 in all cases (see Eq. (4)). The lines are analytical weakdisorder predictions of the asymptotic linear behavior $C_{n} \sim$ $c_{n} L$, taken from reference [26] for $E=0$ and following the RPA for $E=-1$ and $E=-1.95$.

be included, since the central-limit theorem does not yet apply for short distances. This is displayed in Figure 4, which compares $P(\ln \nu)$ for $L=l_{\text {loc }} / 2, E=-1, l_{\text {pert }}=24$ with a normal distribution, which only accounts for $C_{1}^{(\nu)}$ and $C_{2}^{(\nu)}$, and with a generalized normal distribution (the so-called Pearson system [32]),

$$
\begin{aligned}
& P(x)=C\left(a+b x+c x^{2}\right)^{-1 / 2 c} \\
& \quad \times \exp \left[\frac{(b+2 c m) \arctan \left[(b+2 c x) / \sqrt{4 a c-b^{2}}\right]}{c \sqrt{4 a c-b^{2}}}\right],
\end{aligned}
$$

which accounts for the first four cumulants by the four constants $a, b, c$, and $m$. The cumulants have been reconstructed from their asymptotics (1b) (hence, from the asymptotically defined quantities $c_{n}^{(\nu)}$ and $d_{n}^{(\nu)}$ ), and differ from the numerical values of the data by less than three percent. 
(a) $E=0$
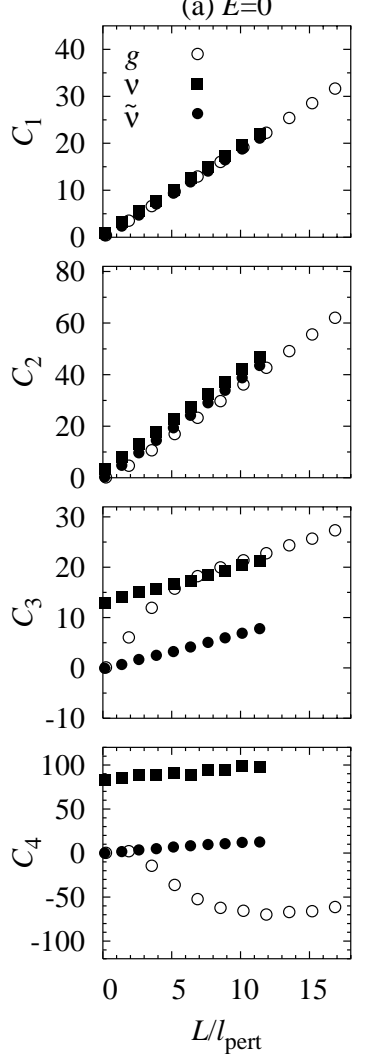

(b) $E=-1$


(c) $E=-1.95$
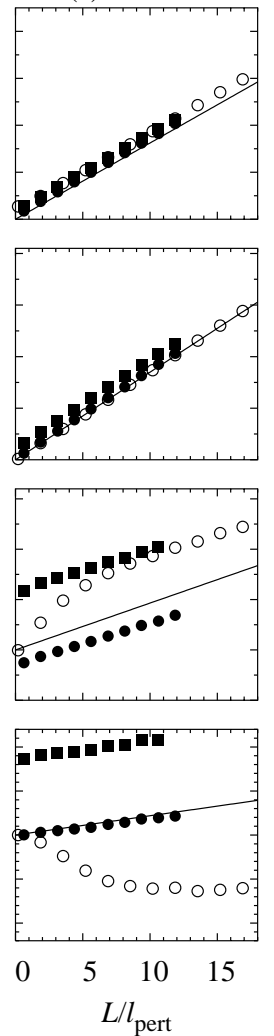

Fig. 2. Same as Figure 1, but for stronger disorder with $l_{\text {pert }}=24: D_{V}=1 / 6$ (left), $D_{V}=1 / 8$ (middle), $D_{V}=0.00823$ (right). The lines in the right panels (c) are the analytic predictions for the given disorder strength close to the band edge, taken from reference [27].

\subsection{Analytical theory for the slopes $c_{n}$ and offsets $d_{n}$}

\subsubsection{Slopes $c_{n}$}

Recently [26,27], we have been able to extent Halperin's phase formalism $[22,33]$, which allows to calculate $l_{\text {loc }}$ and hence $c_{1}$, to all slopes $c_{n}$. This formalism can be applied for arbitrary $\lambda_{\mathrm{F}} / l_{\text {pert }}$, i.e., also for relatively strong disorder, as long as $l_{\text {pert }} \gg 1$ (the lattice constant, set to unity in this paper). Other formalisms like the supersymmetric $\sigma$ model and the Berezinskii technique are rather more restrictive and cannot directly address the logarithm of $g$ and $\nu$. It turned out that three different regions of energy have to be distinguished in the one-dimensional Anderson model. For energies $2-|E| \gtrsim D^{2 / 3}$ close to the band edge, corresponding to relatively strong disorder, the RPA fails and the distribution function deviates from the strict log-normal form [27]. RPA fails also for energies $|E| \lesssim D$ close to the band-center [34], and the distribution function again deviates from the strict log-normal form [26], in generalization of the Kappus-Wegner anomaly of $l_{\text {loc }}$ at $E=0[24,35,36]$. For other energies inside the band, the RPA is justified, and SPS holds, for weak disorder.

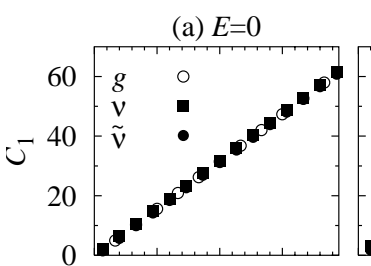

(b) $E=-1$
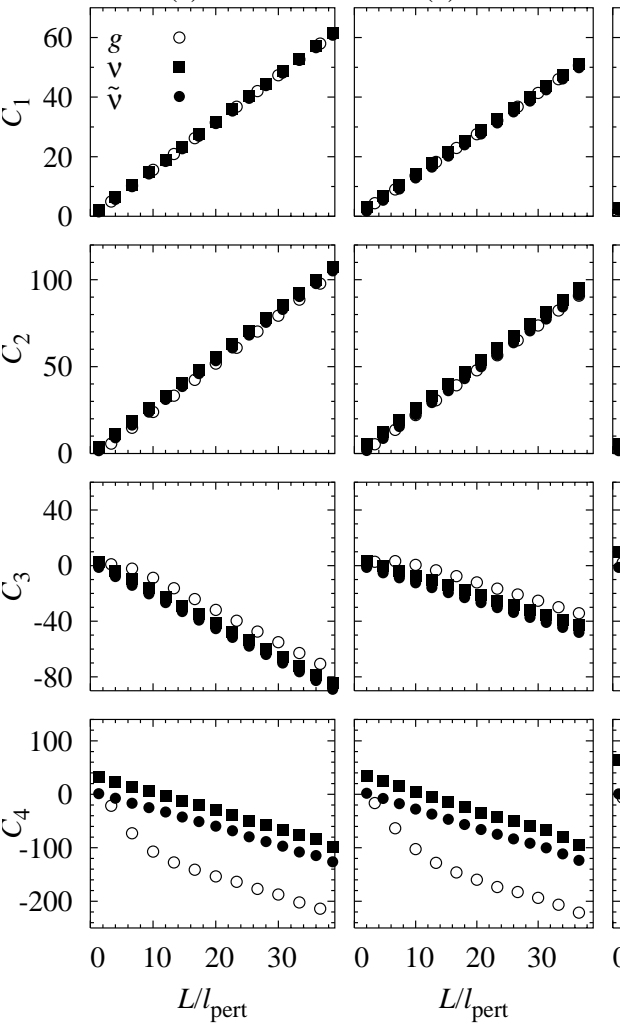

(c) $E=-1.95$
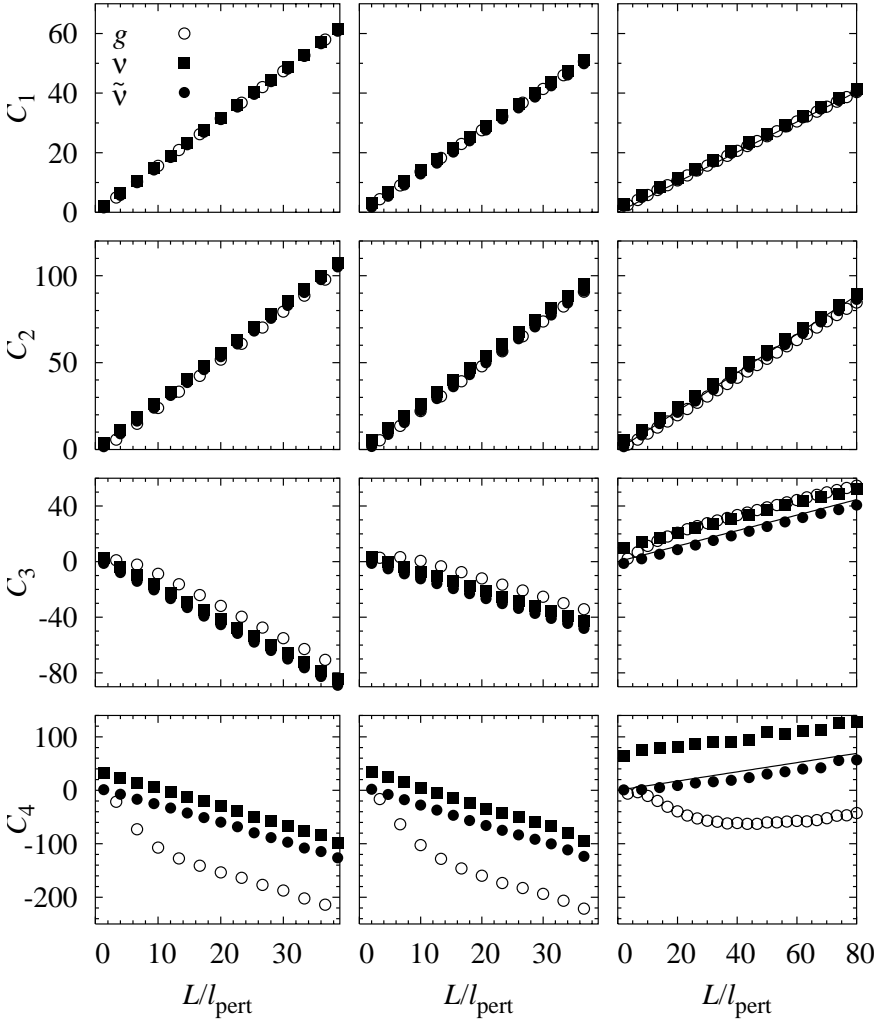

Fig. 3. Same as Figures 1 and 2, but for stronger disorder with $l_{\text {pert }}=1.5: D_{V}=8 / 3$ (left), $D_{V}=2$ (middle), $D_{V}=$ 0.1316 (right). The lines in the right panels (c) are the analytic predictions for the given disorder strength close to the band edge, taken from reference [27].

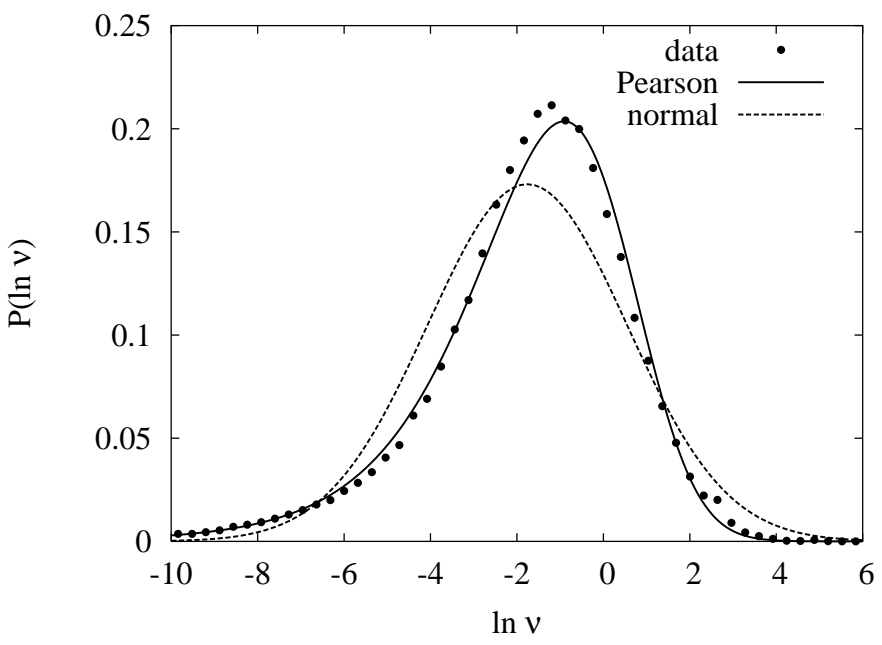

Fig. 4. The probability distribution function $P(\ln \nu)$ from a numerical simulation (data points) in the Anderson model with $L=l_{\text {loc }} / 2, E=-1, l_{\text {pert }}=24$ is compared to a normal distribution (dashed line) with the same mean and variance as the data, and a generalized normal distribution from the Pearson system (solid line), equation (6), where the four free parameters are determined from the asymptotic values of the first four cumulants. 
Offsite disorder adds another means to depart from SPS at the band center, since the balance parameter $\delta$ interpolates between the Kappus-Wegner anomaly at $\delta=$ $1\left(D_{t}=0\right)$ and the Dyson singularity at $\delta=-1\left(D_{V}=0\right)$, which results in total delocalization [37-41]. In the vicinity of the band center, we derive the following Fokker-Planck equation for the joint distribution function $P(u, \alpha ; L)$ of $u \simeq-\ln g \simeq-\ln \nu$, and the phase $\alpha$ for reflection from the system:

$$
\begin{aligned}
\left(D_{V}+D_{t}\right)^{-1} \frac{\partial P}{\partial L}=\left[\partial_{\alpha}\left(\frac{\delta}{2} s_{2 \alpha}-\varepsilon\right)+\partial_{\alpha}^{2}\left(1+\delta c_{\alpha}^{2}\right)\right. \\
\left.-\frac{1}{2} \partial_{u}\left(1+\delta c_{2 \alpha}\right)+\frac{1}{2} \partial_{u}^{2}\left(1-\delta c_{2 \alpha}\right)+\partial_{u} \partial_{\alpha} \delta s_{2 \alpha}\right] P
\end{aligned}
$$

where $s_{x}=\sin x, c_{x}=\cos x, \varepsilon=E /\left(D_{V}+D_{t}\right)$, and $\partial$ denotes partial derivatives. For $\delta=1$, this equation has been used to study the wavefunction statistics at the KappusWegner anomaly [27]. For $\delta=-1, E=0$, one recovers the delocalization at the Dyson anomaly. At the balance point of onsite and offsite disorder $\delta=0$, the variables $u$ and $\alpha$ decouple. For $L \rightarrow \infty$ the reflection phase $\alpha$ becomes completely random, while $P(u)$ precisely takes the form of SPS, with asymptotic slopes $c_{2}=2 c_{1}$ and $c_{n}=0$ for $n \geq 3$. Hence, somewhat surprisingly, we find that RPA and SPS hold true by a particularly simple mechanism just in between the two abovementioned anomalies.

Away from the novel SPS point $\delta=0$, but for disorder still small, the asymptotic behavior of the distribution function can be analyzed by introducing into equation (7) the large-deviation ansatz

$$
P(u, \alpha ; L)=\int_{-i \infty}^{+i \infty} \frac{d \xi}{2 \pi i} \sum_{k=0}^{\infty} \exp [c(\xi) L-\xi u] f(\alpha ; \xi) .
$$

Here $c(\xi)=\sum_{n} \xi^{n} c_{n} / n$ ! is the generating function of the slopes of the cumulants, see equations (1) and (2), and $f(\alpha ; \xi)$ has to be periodic and normalizable with respect to $\alpha$. We arrive at a differential equation

$$
\begin{aligned}
\frac{c(\xi) f(\alpha ; \xi)}{D_{V}+D_{t}} & =\left[\partial_{\alpha}\left(\frac{\delta}{2} s_{2 \alpha}-\varepsilon\right)+\partial_{\alpha}^{2}\left(1+\delta c_{\alpha}^{2}\right)\right. \\
+ & \left.\frac{\xi}{2}\left(1+\delta c_{2 \alpha}-2 \partial_{\alpha} \delta s_{2 \alpha}\right)+\frac{\xi^{2}}{2}\left(1-\delta c_{2 \alpha}\right)\right] f(\alpha ; \xi)
\end{aligned}
$$

in which the slope-generating function $c(\xi)$ appears as an eigenvalue, while $f(\alpha ; \xi)$ appears as an eigenfunction. The slopes $c_{n}$ now can be calculated iteratively by expanding $c(\xi)$ and $f(\alpha ; \xi)$ order by order in $\xi$, following references [26,27]. Away from the SPS point $\delta=0$ but for $|E| \lesssim D_{V}+D_{t}$, the slopes $c_{n}$ take finite values, in compliance with equation (1). Our analytical results are confirmed by numerical computations in Figure 5 .

\subsubsection{Constant offsets $d_{n}$}

The offsets $d_{n}^{(\nu)} \approx C_{n}^{(\nu)}(L=0)$ can be calculated by expressing the local density of states $\nu(L)$ in terms of the re-
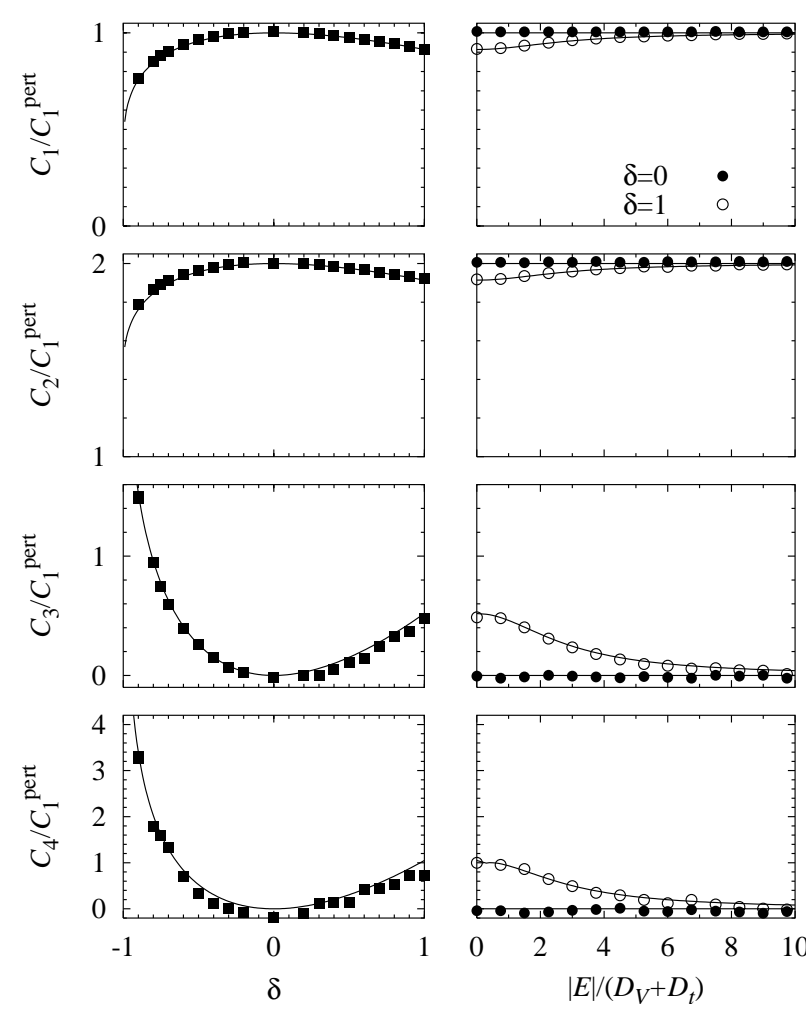

Fig. 5. Cumulants $C_{n}$ (in units of $C_{1}^{\text {pert }}=2 L / l_{\text {pert }}$ ) in the asymptotic limit $L \gg l_{\text {loc }}$, as a function of the balance parameter $\delta$ for energy $E=0$ (left panels), and as a function of $E$ for $\delta=0$ and $\delta=1$ (right panels). Results of numerical simulations with $l_{\text {pert }}=300$ are compared to the predictions of the analytical theory.

flection coefficients $r_{\mathrm{R}}\left(r_{\mathrm{L}}\right)$ from the segment of the wire to the right (left) of the point $L$ at which $\nu$ is calculated [30],

$$
\nu(L)=\operatorname{Re} \frac{\left(1+r_{\mathrm{L}}\right)\left(1+r_{\mathrm{R}}\right)}{1-r_{\mathrm{L}} r_{\mathrm{R}}},
$$

where we normalized $\langle\nu(L)\rangle=1$ (which amounts to multiplication by a constant factor $\pi \sqrt{4-E^{2}}$ ). For $L=0$ and the opening of the wire oriented to the left, $r_{\mathrm{L}}=0$ because there is no reflection from the opening, and $r_{\mathrm{R}}=\exp (i \alpha)$, where $\alpha$ is the phase of reflection from the semi-infinite system. Hence, the numbers

$$
d_{n}^{(\nu)} \approx C_{n}^{(\nu)}(L=0)=\left\langle\left\langle[-\ln (1+\cos \alpha)]^{n}\right\rangle\right\rangle
$$

characterize the distribution of the reflection phase $\alpha$ of the semi-infinite system [30], and allow to assess the validity of the RPA, which predicts $d_{1}^{(\nu)}=\ln 2, d_{2}^{(\nu)}=\pi^{2} / 3$, $d_{3}^{(\nu)}=12 \zeta(3)$ (with the Riemann $\zeta$ function), $d_{4}^{(\nu)}=$ $14 \pi^{4} / 15$.

The offsets $d_{n}^{(\tilde{\nu})} \approx C_{n}^{(\tilde{\nu})}(L=0)$ vanish independently of the RPA since in terms of the reflection matrices introduced above

$$
\tilde{\nu}(L)=\operatorname{Re} \frac{1+r_{\mathrm{L}} r_{\mathrm{R}}}{1-r_{\mathrm{L}} r_{\mathrm{R}}}
$$

and hence $\tilde{\nu}(0)=1$. 
(a) $\delta$ varies, $E=0, l_{\text {pert }}=300$


(b) $\delta=1, E$ varies, $l_{\text {pert }} \gg 1$
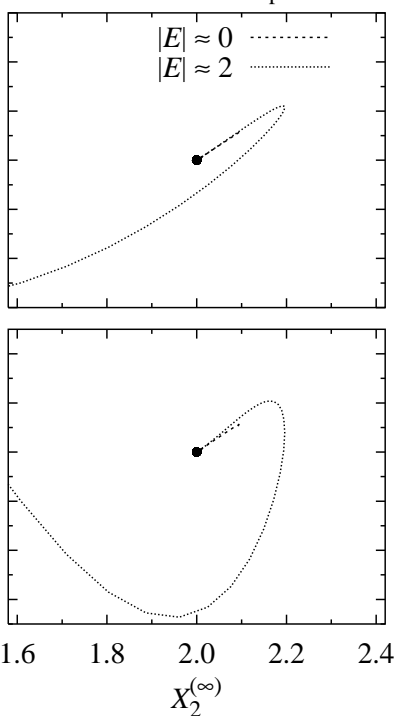

(c) $\delta=1, E$ fixed, $l_{\text {pert }}=1.5-300$
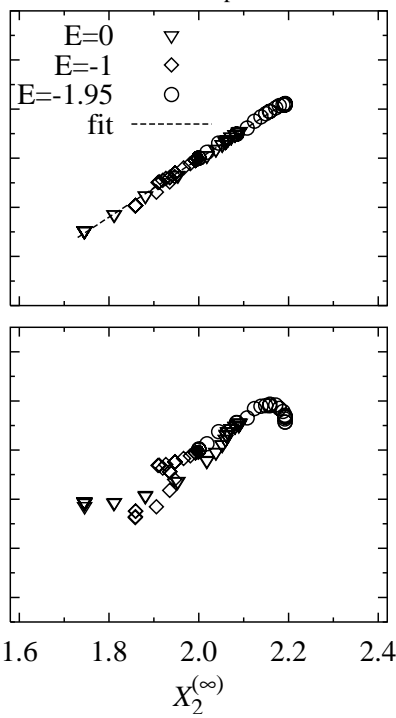

(d) combined results


Fig. 6. Dependence of the parameters $X_{3}^{(\infty)}$ and $X_{4}^{(\infty)}$ on $X_{2}^{(\infty)}$ for various ways to depart from SPS (SPS conditions are indicated by the dot at coordinates $(2,0)$ ). (a) The balance of weak onsite and offsite disorder is changed at the center of the band, $E=0$. The curve is the prediction of the analytical theory, while data points are obtained by numerical simulations. (b) Analytical results as energy is varied at a fixed strength of onsite disorder, with $l_{\text {pert }} \gg 1$ but $l_{\text {pert }} / \lambda_{\mathrm{F}}$ arbitrary, around the band center $(E=0)$ and around the band edge $(|E| \approx 2)$. (c) Numerics and linear fits for variable strength of onsite disorder at three different values of the energy. (d) Comparison of the curves in (a-c).

The offsets $d_{n}^{(g)}$ are obtained by considering the composition law $t_{\mathrm{R}+\mathrm{L}}=t_{\mathrm{R}}\left(1-r_{\mathrm{L}} r_{\mathrm{R}}\right)^{-1} t_{\mathrm{L}}$ for the series transmission through two long segments $\mathrm{R}$ and $\mathrm{L}$. The reflection coefficients now are equivalent phase factors $r_{\mathrm{R}, \mathrm{L}}=\exp \left(i \alpha_{\mathrm{R}, \mathrm{L}}\right)$. We equate the cumulants of both sides and insert the asymptotics (1). The constant offsets follow as $d_{n}^{(g)}=(-1)^{n}\left\langle\left\langle\left\{\ln \left[2-2 \cos \left(\alpha_{\mathrm{R}}+\alpha_{\mathrm{L}}\right)\right]\right\}^{n}\right\rangle\right\rangle$. In the RPA, $d_{1}^{(g)}=0$ and $d_{n}^{(g)}=(-1)^{n} d_{n}^{(\nu)}$. This is clearly displayed in Figure 1. Beyond the RPA, the $d_{n}^{(g)}$ and $d_{n}^{(\nu)}$ contain equivalent information on the reflection-phase distribution function $P(\alpha)$, but no longer are simply related.

\subsection{Independence of the parameters}

Now we turn to the question of the mutual independence of the parameters $c_{n}$, as we violate the conditions for SPS.

A convenient set of parameters beyond the SPS quantity $C_{1}$ is formed by the ratios $X_{n}=C_{n} / C_{1}$, which asymptotically acquire the constant values

$$
\lim _{L \rightarrow \infty} X_{n}=X_{n}^{(\infty)}=c_{n} / c_{1} .
$$

In RPA and SPS, only one effective parameter $C_{1}$ survives since $c_{2}^{(g)}=c_{2}^{(\nu)}=2 c_{1}^{(g)}=2 c_{1}^{(\nu)}$, i.e., $X_{2}^{(\infty)}=2$, and moreover $c_{n}^{(g)}=c_{n}^{(\nu)}=0$ for $n \geq 3$, which gives a picture consistent with SPS. However, beyond this approximation the cumulants $C_{n}$ with $n \geq 3$ generally may increase linearly with $L$, and hence can be of the same order as $C_{1}$ and $C_{2}$, such that all $X_{n}^{(\infty)}$ are of order unity. Notice that the asymptotic value $X_{n}^{(\infty)}$ is well approximated by $X_{n}^{(\tilde{\nu})}$ even for $L \lesssim l_{\text {loc }}$, since the cumulants $C_{n}^{(\tilde{\nu})}$ are linear already for small $L$ and the offsets $d_{n}^{(\tilde{\nu})}$ vanish.

In Figure 6 we plot the asymptotic ratios of cumulants $X_{3}^{(\infty)}$ and $X_{4}^{(\infty)}$ as function of $X_{2}^{(\infty)}$, while we vary:

(a) the balance parameter $\delta$ at $E=0$ (a i) from 0 to 1 and (a ii) from 0 to -1 ;

(b) energy for fixed onsite disorder (b i) around $E=0$ and (b ii) around $|E|=2$; and

(c) the disorder strength from $l_{\text {pert }}=300$ to $l_{\text {pert }}=1.5$ at $\delta=1$ for the three values of energy (c i) $E=0$, (c ii) $E=-1$, and (c iii) $E=-1.95$.

In the cases (a) and (b) we show the results of the analytical procedure described above, while for (c) we show the result of the numerical simulations. For illustration of the predictive power of the theory presented in the previous Section 3.2, numerical results are also displayed for case (a).

Of particular interest is the curve for case (b ii), for energies close to the band edge, which also applies to strong disorder, $D^{2 / 3} \gtrsim 2-|E|[27]$. (See also the data points for case (c iii).) In this case the curves $X_{n}^{(\infty)}\left(X_{2}^{(\infty)}\right)$ depart from the seemingly unique functional behavior obtained in the other cases. Hence, we are led to conclude that at least for sufficiently strong disorder $X_{3}^{(\infty)}$ and $X_{4}^{(\infty)}$ are not uniquely determined by $X_{2}^{(\infty)}$. Since the SPS quantity $C_{1}$ is always an independent scaling parameter, altogether more than two quantities are needed to characterize the distribution function $P(\nu)$ for short distances $L \lesssim l_{\text {loc }}$ (where the central-limit theorem, and hence TPS, not yet applies). 


\section{Conclusions}

We observed that the short-distance statistics of localized wavefunctions inside a long one-dimensional disordered system can be recovered from the long-distance statistics, but in general are characterized by more than the two parameters (a mean $C_{1}$ and a variance $C_{2}$ ) that suffice to describe the long-distance statistics themselves. These additional parameters have been obtained from the higher cumulants $C_{n}$ of $\ln \nu$, where $\nu$ is the local density in a semi-infinite system. The additional parameters in the $C_{n}$ can be neglected when considering the case of weak disorder and generic energies within the band: Then $C_{1} \sim C_{2} / 2 \propto L$, and also $C_{n}=O\left(L^{0}\right)$ for $n \geq 3$ take universal values, which results in a picture consistent with single-parameter scaling even in the short-distance wavefunction statistics.

With three-dimensional systems in mind, it would be desirable to investigate the relation of the parameters from large deviation statistics to the scaling parameters at the metal-insulator transition, which may be established by multi-fractal analysis when this transition is approached from the localized regime.

Another potential application of the higher cumulants is to use them for detecting spatial correlations in the disorder, since the higher cumulants are sensitive to morepoint wavefunction correlations. This offers a natural extension of a previous investigation [42], which demonstrated that deviations from randomness due to spatial three-point correlations (such as displayed by a folded Fibonacci sequence) cannot be detected by the conventional wavefunction statistics.

\section{References}

1. P. Sheng, Scattering and Localization of Classical Waves in Random Media (World Scientific, Singapore, 1990)

2. R. Berkovits, S. Feng, Phys. Rep. 238, 135 (1994)

3. P.W. Anderson, Phys. Rev. 109, 1492 (1958)

4. P.A. Lee, T.V. Ramakrishnan, Rev. Mod. Phys. 57, 287 (1985)

5. B. Kramer, A. MacKinnon, Rep. Prog. Phys. 56, 1469 (1993)

6. C.W.J. Beenakker, Rev. Mod. Phys. 69, 731 (1997)

7. M. Janssen, Phys. Rep. 295, 1 (1998)

8. A.D. Mirlin, Phys. Rep. 326, 259 (2000)

9. A. Cohen, Y. Roth, B. Shapiro, Phys. Rev. B 38, 12125 (1988)

10. P. Markoš, B. Kramer, Phil. Mag. B 68, 357 (1993); Ann. Physik (Leipzig) 2, 339 (1993)

11. L.I. Deych, D. Zaslavsky, A.A. Lisyansky, Phys. Rev. Lett. 81, 5390 (1998)

12. L.I. Deych, A.A. Lisyansky, B.L. Altshuler, Phys. Rev. Lett. 84, 2678 (2000); Phys. Rev. B 64, 224202 (2001)
13. L.I. Deych, M.V. Erementchouk, A.A. Lisyansky, Phys. Rev. Lett. 90, 126601 (2003); Phys. Rev. B 67, 024205 (2003)

14. P.-G. Luan, Z. Ye, Phys. Rev. E 64, 066609 (2001)

15. J.W. Kantelhardt, A. Bunde, Phys. Rev. B 66, 035118 (2002)

16. S.L.A. de Queiroz, Phys. Rev. B 66, 195113 (2002)

17. E. Abrahams, P.W. Anderson, D.C. Licciardello, T.V. Ramakrishnan, Phys. Rev. Lett. 42, 673 (1979)

18. P.W. Anderson, D.J. Thouless, E. Abrahams, D.S. Fisher, Phys. Rev. B 22, 3519 (1980)

19. B.L. Altshuler, V.E. Kravtsov, I.V. Lerner, Pis'ma Zh. Eksp. Teor. Fiz. 43, 342 (1986) [JETP Lett. 43, 441 (1986)]; Zh. Eksp. Teor. Fiz. 91, 2276 (1986) [Sov. Phys. JETP 64, 1352 (1986)]

20. K. Slevin, P. Markos, T. Ohtsuki, Phys. Rev. Lett. 86, 3594 (2001); Phys. Rev. B 67, 155106 (2003)

21. A.A. Abrikosov, Solid State Commun. 37, 997 (1981)

22. I.M. Lifshitz, S.A. Gredeskul, L.A. Pastur, Introduction to the Theory of Disordered Systems (Wiley, New York, 1988)

23. B.L. Altshuler, V.N. Prigodin, Zh. Eksp. Teor. Fiz. 95, 348 (1989) [Sov. Phys. JETP 68, 198 (1989)]

24. M. Kappus, F. Wegner, Z. Phys. B 45, 15 (1981)

25. R.S. Ellis, Entropy, Large Deviations and Statistical Mechanics (Springer, New York, 1985)

26. H. Schomerus, M. Titov, Phys. Rev. B 67, 100201(R) (2003)

27. H. Schomerus, M. Titov, Phys. Rev. E 66, 066207 (2002)

28. V.I. Oseledec, Trudy Moskov. Mat. Obshch. 19, 179 (1968) [T. Moscow Math. Soc. 19, 197 (1968)]

29. P.J. Roberts, J. Phys.: Condens. Matter 4, 7795 (1992)

30. H. Schomerus, M. Titov, P.W. Brouwer, C.W.J. Beenakker, Phys. Rev. B 65, 121101(R) (2002)

31. D.J. Thouless, in Ill-Condensed Matter, edited by R. Balian, R. Maynard, G. Toulouse (North-Holland, Amsterdam, 1979)

32. K. Pearson, Phil. Trans. A 216, 429, (1916); C.C. Craig, Ann. Math. Stat. 7, 16 (1936); C. Craig, in Mathematics of Statistics Pt. 2, edited by J.F. Kenney, E.S. Keeping (Van Nostrand, Princeton, NJ, 1951), p. 107

33. B.I. Halperin, Phys. Rev. B 139A, 104 (1965)

34. A.D. Stone, D.C. Allan, J.D. Joannopoulos, Phys. Rev. B 27, 836 (1983)

35. B. Derrida, E. Gardner, J. Phys. France 45, 1283 (1984)

36. I. Goldhirsch, S.H. Noskowicz, Z. Schuss, Phys. Rev. B 49, 14504 (1994)

37. G. Theodorou, M.H. Cohen, Phys. Rev. B 13, 4597 (1976)

38. T.P. Eggarter, R. Riedinger, Phys. Rev. B 18, 569 (1978)

39. A.A. Gogolin, V.I. Melnikov, Zh. Eksp. Teor. Fiz. 73, 706 (1977) [Sov. Phys. JETP 46, 369 (1977)]

40. A.A. Gogolin, Zh. Eksp. Teor. Fiz. 77, 1649 (1979) [Sov. Phys. JETP 50, 827 (1979)]

41. A. Bovier, J. Stat. Phys. 56, 645 (1989)

42. N. Brenner, S. Fishman, Nonlinearity 5, 211 (1992) 